\title{
Self-consistent Ornstein-Zernike approximation for three-dimensional spins
}

\author{
D. Pini \\ Istituto Nazionale per la Fisica della Materia e \\ Università degli Studi di Milano, via Celoria 16, 20133 Milano, Italy \\ J. S. Høye \\ Institutt for Fysikk, NTNU, \\ N 7491 Trondheim, Norway. \\ G. Stell \\ Department of Chemistry, State University of New York at Stony Brook, \\ Stony Brook, NY 11794-3400, U.S.A.
}

\begin{abstract}
An Ornstein-Zernike approximation for the two-body correlation function embodying thermodynamic consistency is applied to a system of classical Heisenberg spins on a three-dimensional lattice. The consistency condition determined in a previous work is supplemented by introducing a simplified expression for the meansquare fluctuations of the spin on each lattice site. The thermodynamics and the correlations obtained by this closure are then compared with approximants based on extrapolation of series expansions and with Monte Carlo simulations. The comparison reveals that many properties of the model, including the critical temperature, are very well reproduced by this simple version of the theory, but that it shows substantial quantitative error in the critical region, both above the critical temperature and with respect to its rendering of the spontaneous magnetization curve. A less simple but conceptually more satisfactory version of the SCOZA is then developed, but not solved, in which the effects of transverse correlations on the longitudinal susceptibility is included, yielding a more complete and accurate description of the spin-wave properties of the model.
\end{abstract}

\section{Introduction}

Several approaches in the context of liquid-state theory hinge on the Ornstein-Zernike (OZ) equation that relates the two-body distribution function $g(\mathbf{r})$ and the direct correla- 
tion function $c(\mathbf{r})$. Once an approximate expression for $c(\mathbf{r})$ is inserted into this equation, a closed theory for the thermodynamics and the correlations is obtained.

While the dependence of $c(\mathbf{r})$ on the thermodynamic variables is fed directly into the expression of $c(\mathbf{r})$ itself in the commonly used closures to the OZ equation, another method for obtaining a closed theory amounts to requiring that $c(\mathbf{r})$ depends on the density and on the temperature of the system in such a way that consistency between different paths to the thermodynamics is obtained. Recent results on simple lattice gases [1] and model fluids [2] have shown that such a self-consistent Ornstein-Zernike approximation (SCOZA) is able to yield very good results for both the overall thermodynamics and correlations and for the critical point and the coexistence curve.

The idea of using thermodynamic consistency as a constraint to close the OZ equation is relevant not only to simple fluids. In particular, the theory has already been formulated also for the $D$-vector model [3], i.e., a system of classical $D$-dimensional spins with nearest-neighbor interaction. In the present paper the $D$-vector model SCOZA is further investigated and the simplest version of the SCOZA that we have developed is solved numerically in the case of three-dimensional spins (classical Heisenberg model) on a threedimensional lattice, and the results are compared with Monte Carlo simulations [ [4, 5, 6] and Padè approximants based on high-temperature expansions [7, 8, 9, 10, 11]. An analysis of what is lacking in this simple application to the $D$-vector model then guides us in formulating a more sophisticated version of the theory that takes into account the influence of transverse correlations on the longitudinal susceptibility and as a result, yields a more complete and accurate description of the spin-wave properties of the model.

Besides allowing us to test the SCOZA for a system with a vector order parameter, we believe that the investigation set forth here is also interesting in its own sake. While the main properties of the D-vector model have been understood for a long time and its critical constants have been determined with great accuracy in several recent papers both by finite-size scaling techniques [6, 5] and by extrapolation of series expansions [11], there is nevertheless a paucity of treatments that yield a reasonable description of the model for $D=3$ (the classical Heisenberg model) over the whole phase plane, especially compared to the Ising or $D=1$ case. The $D$-vector model has recently been studied by cumulant expansion methods [12, 13], which can be regarded as a systematic way to improve the mean-field approximation. However, results were determined only in zero magnetic field, and do not include the correlation functions. Moreover, the predictions for the critical temperature obtained within the currently adopted truncation of the cumulant expansion are in only modest agreement with the expected values [13]. It seems therefore interesting to develop a version of SCOZA for this model, particularly for $D=2$ and $D=3$, that promises to yield high overall accuracy, especially since our approach gives both the thermodynamics and the correlations of the system.

A difference that arises in the present implementation of the SCOZA with respect to the Ising case is that the $\mathrm{OZ}$ approximation (i.e., the assumption that the direct correlation function $c(\mathbf{r})$ has the same range as the interaction between the spins) and the shortrange constraint on the two-body correlation function which stems from the fixed length of the spins are not enough to get a closed theory by means of a single thermodynamic 
consistency condition. We need some ansatz about the mean-square fluctuations of the transverse and longitudinal spin components. The initial choice we investigate here is to regard these fluctuations of the spin on each lattice site as a function of the magnetization alone, thereby using at every temperature the expression valid in the high-temperature limit. We find that within this approximation the error in the critical temperature is at most about $0.5 \%$, and the compressibility, the correlation length, and the correlation functions are nicely reproduced over a wide range of thermodynamic states. Nevertheless, the global accuracy of this version of the theory is inferior to that of the Ising or simple fluid case. In particular, for both the Ising and the Heisenberg model the SCOZA gives spherical critical exponents above the critical temperature $T_{c}$, but in the Ising case [1] the asymptotic critical regime is detectable only very close to the critical point and the thermodynamics is well reproduced up to reduced temperatures $t=\left(T-T_{c}\right) / T_{c} \sim 10^{-2}$. For the Heisenberg model, on the other hand, the deviations from the correct critical behavior appear already for $t \sim 10^{-1}$. At smaller values of $t$, the theory readily reaches the asymptotic spherical regime. Moreover, while in the Ising-model SCOZA the coexistence curve displays a non-classical exponent $\beta=7 / 20$ [1, 14, the Heisenberg coexistence curve predicted by the SCOZA instead is associated with $\beta=1 / 2$, and is overall considerably narrower than the expected one as given by $\mathrm{MC}$ simulations [⿴囗十 and Padè approximant techniques [8]. (We find however that it displays the correct behavior in the low-temperature limit). In order to probe the sensitiveness of the theory to the approximate ansatz adopted for the mean-square spin fluctuations, we also implemented a different approximation, which was originally proposed in Ref. [3]. The results are almost identical in the two cases. In response to the lack of high quantitative accuracy shown by these simple versions of the theory we then develop (but do not solve here) an improved version that incorporates a more complete and accurate description of spin-wave correlations.

The paper is organized as follows: in Sec. 2 we review the theory already developed in Ref. [3], introduce an approximation necessary to obtain a closed equation, and cast it into a form amenable to numerical solution, which we obtain in Sec. 3. There we show our results for the critical point, the thermodynamics, the correlations, and the phase diagram, and compare them to predictions from MC simulations and Padè approximants. We give our improved version of the theory in Sec. 4 , in which we include the effects of transverse correlations on the longitudinal susceptibility. Our findings are briefly summarized in Sec. 5 .

\section{Theory}

We consider a system of $D$-dimensional vectors $\mathbf{s}_{\mathbf{r}}$ of unit length on a three-dimensional lattice, $\mathbf{r}$ being the position of a generic lattice site, interacting via a nearest-neighbor, ferromagnetic coupling. Here we are interested in the classical analog of the Heisenberg 
model, and therefore will deal with the case $D=3$. The hamiltonian of the system is

$$
\mathcal{H}=-J \sum_{\left\langle\mathbf{r}, \mathbf{r}^{\prime}\right\rangle} \mathbf{s}_{\mathbf{r}} \cdot \mathbf{S}_{\mathbf{r}^{\prime}}-\mathbf{H} \cdot \sum_{\mathbf{r}} \mathbf{s}_{\mathbf{r}}
$$

where the sum over $\mathbf{r}$ and $\mathbf{r}^{\prime}$ extends over all nearest-neighbor couples, $\mathbf{H}$ is the external magnetic field, and $J>0$ is the coupling constant, which will be taken equal to unity in the following. The SCOZA for this model was originally introduced in Ref. [3]. We begin by reviewing it in some detail.

The reduced longitudinal and transverse isothermal susceptibilities $\chi_{\|}, \chi_{\perp}$ are defined by:

$$
\begin{aligned}
\frac{1}{\chi_{\|}} & =\left.\left(\frac{\partial \beta H_{\|}}{\partial m_{\|}}\right)_{\beta}\right|_{m_{\|}=m}, \\
\frac{1}{\chi_{\perp}} & =\left.\left(\frac{\partial \beta H_{\perp}}{\partial m_{\perp}}\right)_{\beta}\right|_{m_{\perp=0}},
\end{aligned}
$$

where $m$ is the magnetization per spin and $\beta=1 / k_{\mathrm{B}} T, T$ being the absolute temperature, and $k_{\mathrm{B}}$ the Boltzmann's constant. (We remark that the definitions of $\chi_{\|}, \chi_{\perp}$ used here differ from those in Ref. [3] by a factor $\beta$ ). Here the symbols $\|$ and $\perp$ refer respectively to the direction along the magnetic field $\mathbf{H}$, and to any of the $D-1$ directions of the coordinate axes lying in the plane perpendicular to $\mathbf{H}$. Because of the spherical symmetry of the system, we find

$$
\begin{aligned}
& \frac{1}{\chi_{\|}}=\left(\frac{\partial \beta H}{\partial m}\right)_{\beta}=\left(\frac{\partial^{2} \beta a}{\partial m^{2}}\right)_{\beta}, \\
& \frac{1}{\chi_{\perp}}=\frac{\beta H}{m}=\frac{1}{m}\left(\frac{\partial \beta a}{\partial m}\right)_{\beta},
\end{aligned}
$$

where $a=a(m, \beta)$ is the Helmholtz free energy per spin. Let us note that Eqs. (4), (5) imply

$$
\frac{1}{\chi_{\|}}=\frac{\partial}{\partial m}\left(\frac{m}{\chi_{\perp}}\right)
$$

The internal energy per spin $u$ is given by

$$
u=\left(\frac{\partial \beta a}{\partial \beta}\right)_{m} .
$$

We now introduce the two-spin correlation function matrix $\boldsymbol{\Gamma}(\mathbf{r})$ with elements

$$
\Gamma_{i j}(\mathbf{r})=\left\langle s_{\mathbf{r}}^{i} s_{\mathbf{0}}^{j}\right\rangle-\left\langle s_{\mathbf{r}}^{i}\right\rangle\left\langle s_{\mathbf{0}}^{j}\right\rangle,
$$


where the indexes $i, j$ refer to two generic components of the vectors $\mathbf{s}_{\mathbf{r}}, \mathbf{s}_{\mathbf{0}}$. Isotropy implies that $\boldsymbol{\Gamma}$ can be put into diagonal form by choosing the direction of the magnetic field $\mathbf{H}$ as one of the coordinate axis. In particular, we have

$$
\begin{aligned}
\Gamma_{\|}(\mathbf{r}) & =\left\langle s_{\mathbf{r}}^{\|} s_{\mathbf{0}}^{\|}\right\rangle-m^{2}, \\
\Gamma_{\perp}(\mathbf{r}) & =\left\langle s_{\mathbf{r}}^{\perp} s_{\mathbf{0}}^{\perp}\right\rangle .
\end{aligned}
$$

We recall that both the internal energy $u$ and the susceptibilities $\chi_{\|}, \chi_{\perp}$ can be expressed by the correlation functions $\Gamma_{\|}, \Gamma_{\perp}$. Specifically, we have for the internal energy

$$
u=-\frac{q}{2}\left[m^{2}+\Gamma_{\|}(\boldsymbol{\lambda})+d \Gamma_{\perp}(\boldsymbol{\lambda})\right]
$$

Here $\boldsymbol{\lambda}$ is a vector joining a given lattice site with any of its nearest neighbors, $q$ is the number of nearest neighbors, and $d=D-1$. (In Ref. [3] the factor $q$ is not used. This corresponds to setting $J q=1$ ). The susceptibilities are given by

$$
\begin{aligned}
& \chi_{\|}=\hat{\Gamma}_{\|}(k=0), \\
& \chi_{\perp}=\hat{\Gamma}_{\perp}(k=0),
\end{aligned}
$$

where $\hat{\Gamma}_{\|}(\mathbf{k}), \hat{\Gamma}_{\perp}(\mathbf{k})$ are the Fourier transforms of $\Gamma_{\|}(\mathbf{r}), \Gamma_{\perp}(\mathbf{r})$. Since the length of each spin is equal to unity, we have for every site $\mathbf{r}$

$$
\left\langle\left(s_{\mathbf{r}}^{\|}\right)^{2}\right\rangle+d\left\langle\left(s_{\mathbf{r}}^{\perp}\right)^{2}\right\rangle=1 .
$$

If we set

$$
\begin{aligned}
& R_{\|} \equiv \Gamma_{\|}(r=0), \\
& R_{\perp} \equiv \Gamma_{\perp}(r=0),
\end{aligned}
$$

we find from Eqs. (9), (10) that the condition (14) becomes

$$
R_{\|}+d R_{\perp}=1-m^{2}
$$

Let us now consider the direct correlation function $c(\mathbf{r})$ whose Fourier transform $\hat{c}(\mathbf{k})$ is related to $\hat{\Gamma}(\mathbf{k})$ by

$$
\Gamma(\mathbf{k})=\frac{R}{1-R \hat{c}(\mathbf{k})},
$$

where we have understood the subscripts $\|, \perp$ in both $\hat{\Gamma}(\mathbf{k})$ and $\hat{c}(\mathbf{k})$. In order to simplify the notation, we will adopt this same convention in the remaining of the Section whenever a relation applies to both the longitudinal and the transverse components of the correlation functions. Eq. (18) corresponds to the OZ equation commonly used in liquid-state theory [15. We note that, since the matrix $\Gamma(\mathbf{r})$ is diagonal, Eq. (18) is a purely scalar relation. Here we use the ansatz also known as $\mathrm{OZ}$ approximation, namely we assume 
that $c(\mathbf{r})$ has the same range as the interaction between the spins. For the model in study we have then

$$
c(\mathbf{r})= \begin{cases}c_{0} & \mathbf{r}=0 \\ c_{1} & \mathbf{r}=\boldsymbol{\lambda} \\ 0 & \text { otherwise }\end{cases}
$$

Eq. (19) is readily written in Fourier space:

$$
\hat{c}(\mathbf{k})=c_{0}+q c_{1} \gamma(\mathbf{k})
$$

where the nearest-neighbor Fourier transform $\gamma(\mathbf{k})$ is given by

$$
\gamma(\mathbf{k})=\frac{1}{q} \sum_{\lambda=\text { n.n. }} \mathrm{e}^{-i \mathbf{k} \cdot \lambda}
$$

the sum running over all the nearest neighbors of a given lattice site. We can now use the OZ equation (18) to express $\Gamma(\mathbf{r})$ via $\hat{c}(\mathbf{k})$. Let us introduce the quantity $z$ given by

$$
z=\frac{q R c_{1}}{1-R c_{0}}
$$

and the lattice Green's function

$$
P(z)=\int_{\mathcal{B}} \frac{d^{3} \mathbf{k}}{(2 \pi)^{3}} \frac{1}{1-z \gamma(\mathbf{k})},
$$

where $\mathcal{B}$ is the first Brillouin zone of the lattice. The function $P(z)$ has been widely studied in the literature [16, 17]. For the simple cubic (SC), body-centered cubic (BCC), and face-centered cubic (FCC) lattices considered here it can be evaluated in terms of elliptic integrals [17]. ¿From Eqs. (15)-(23) we readily find

$$
\begin{aligned}
& c_{0}=\frac{1-P(z)}{R}, \\
& c_{1}=\frac{z P(z)}{q R}, \\
& \Gamma(\boldsymbol{\lambda})=-R \frac{1-P(z)}{z P(z)} .
\end{aligned}
$$

The susceptibilities and the internal energy can then be expressed in terms of $z$ and $R$. From Eqs. (12), (13), (18), (24), (25) we get

$$
\frac{1}{\chi}=\frac{(1-z) P(z)}{R}
$$

while Eqs. (11) and (26) yield

$$
u=\frac{q}{2}\left[-m^{2}+R_{\|} \frac{1-P\left(z_{\|}\right)}{z_{\|} P\left(z_{\|}\right)}+d R_{\perp} \frac{1-P\left(z_{\perp}\right)}{z_{\perp} P\left(z_{\perp}\right)}\right] .
$$


It appears then that within the OZ approximation the thermodynamics and the correlations are determined by the quantities $z_{\|}, z_{\perp}, R_{\|}, R_{\perp}$. These quantities are not all independent: in fact, they must be chosen in such a way that the conditions (6) and (17) are satisfied. As noted above, the former is a consequence of the isotropy of the system, while the latter stems from the requirement of each spin having unit length. The other constraint we want to be satisfied is that of consistency between different routes to the thermodynamics which is peculiar to the SCOZA. According to the energy route, the thermodynamics is determined from the internal energy of the system as given by Eq. (11). On the other hand, knowledge of the correlations may be used to obtain the thermodynamics also via Eq. (12) or (13) for the susceptibilities. This is the analog of the compressibility route in fluid systems. While in a hypotetical exact treatment both routes are obviously bound to yield the the same result, this is general no longer true as soon as approximations are introduced. As is readily found from Eqs. (47), (5), (7), the requirement that the susceptibilities $\chi_{\|}, \chi_{\perp}$ and the internal energy $u$ come from a unique Helmholtz free energy is embodied in the relations

$$
\begin{aligned}
\frac{\partial}{\partial \beta}\left(\frac{1}{\chi_{\|}}\right) & =\frac{\partial^{2} u}{\partial m^{2}} \\
\frac{\partial}{\partial \beta}\left(\frac{1}{\chi_{\perp}}\right) & =\frac{1}{m} \frac{\partial u}{\partial m} .
\end{aligned}
$$

We note that these expression are not independent as they are related by Eq. (6). Substitution of Eqs. (27), (28) into Eq. (30) yields the thermodynamic consistency condition

$$
\frac{1}{q} \frac{\partial}{\partial \beta}\left[\frac{\left(1-z_{\perp}\right) P\left(z_{\perp}\right)}{R_{\perp}}\right]=-1+\frac{1}{2 m} \frac{\partial}{\partial m}\left[R_{\|} \frac{1-P\left(z_{\|}\right)}{z_{\|} P\left(z_{\|}\right)}+d R_{\perp} \frac{1-P\left(z_{\perp}\right)}{z_{\perp} P\left(z_{\perp}\right)}\right],
$$

and there will be a corresponding equation obtained from Eq. (29). Eqs. (6), (17), and (31) are a set of three independent conditions for $z_{\|}, z_{\perp}, R_{\|}, R_{\perp}$. As we have four unknown quantities, this is clearly not enough to obtain a closed set of equations. We then need one more equation, which will be introduced as a further approximate ansatz besides the OZ approximation (19). This is different from what is found in the Ising model or $D=1$ SCOZA, in which the OZ ansatz is the sole approximation introduced in the theory. The closure relation suggested in Ref. [3] was

$$
c_{1}^{\|}=c_{1}^{\perp}
$$

i.e., the nearest-neighbor contributions to the direct correlation function in the longitudinal and in the transverse directions are assumed to coincide. Eq. (25) then gives

$$
R_{\perp}=R_{\|} \frac{z_{\perp} P\left(z_{\perp}\right)}{z_{\|} P\left(z_{\|}\right)} .
$$

On the other hand, Eq. (27) shows that a divergence of the longitudinal or transverse susceptibility corresponds respectively to $z_{\|}=1$ or $z_{\perp}=1$. As is well known [18], in the $D$ vector model on a three-dimensional lattice with $D \geq 2$ both susceptibilities are expected 
to diverge on the spontaneous magnetization curve. If this is actually the case also in the present approximation, then Eq. (33) would imply that on the spontaneous magnetization curve the longitudinal and transverse mean-square deviations of the spin coincide. This seems quite unphysical, especially at low temperature, when one expects the behavior of the system to be governed by small transverse oscillations around the direction of the average magnetization $m$ [19], thereby yielding $R_{\|} \ll R_{\perp}$. As a consequence, although we did implement the approximation of Eq. (32) on order to probe the sensitiveness of the SCOZA to the closure adopted (see Sec. 3), we decided to resort to a different one for most of the computations performed here. Specifically, we simply disregarded the dependence of $R_{\|}, R_{\perp}$ on the temperature of the system, i.e. we took for these quantities the same espressions one finds in the high-temperature limit. Since in this limit the spins on different sites are uncorrelated, it is found from Eqs. (12), (13) that the present approximation amounts to assuming, both for the parallel and the transverse components of $R$,

$$
R=\chi(m, \beta=0) .
$$

The reduced susceptibilities in the high-temperature limit are just those of the noninteracting spin system. They are then straightforwardly determined from the Langevin function:

$$
m_{\mathrm{id}}=\frac{1}{\tanh (\beta H)}-\frac{1}{\beta H},
$$

where the subscript "id" refers to the non-interacting system. Eq. (35) is then inverted to express $R_{\|}, R_{\perp}$ as a function of $m$. We observe that in the approximation of Eq. (34) the condition (17) is trivially satisfied at every temperature. Eqs. (6) and (34) finally allow one to obtain a closed partial differential equation (PDE) for the quantity $z_{\perp}$ from the thermodynamic consistency condition (31). We now perform some manipulations on this equation in order to cast it into a form suitable for numerical integration. Let us note that Eqs. (25), (27) imply $0 \leq z \leq 1$. In this interval the function

$$
y=(1-z) P(z)
$$

is invertible. We then introduce its inverse function $z=F(y), 0 \leq y \leq 1$, and we set

$$
G(y) \equiv \frac{1-y-F(y)}{y F(y)}=\frac{1-P(z)}{z P(z)}
$$

so that Eq. (31) becomes

$$
\frac{1}{q R_{\perp}} \frac{\partial y_{\perp}}{\partial \beta}=-1+\frac{1}{2 m} \frac{\partial}{\partial m}\left[R_{\|} G\left(y_{\|}\right)+d R_{\perp} G\left(y_{\perp}\right)\right]
$$

We now use Eq. (6) along with Eqs. (27), (36) to express $y_{\|}$in terms of $y_{\perp}$ :

$$
y_{\|}=R_{\|} \frac{\partial}{\partial m}\left(m \frac{y_{\perp}}{R_{\perp}}\right) .
$$


In the present approximation in which $R_{\|}, R_{\perp}$ coincide with the longitudinal and transverse susceptibilities of the non-interacting system, it is readily found using Eq. (6) that Eq. (39) becomes

$$
y_{\|}=y_{\perp}+m \frac{R_{\|}}{R_{\perp}} \frac{\partial y_{\perp}}{\partial m} .
$$

Moreover, we find

$$
\frac{\partial y_{\|}}{\partial m}=\left(2+\frac{m}{R_{\perp}} R_{\|}^{\prime}\right) \frac{\partial y_{\perp}}{\partial m}+m \frac{R_{\|}}{R_{\perp}} \frac{\partial^{2} y_{\perp}}{\partial m^{2}}
$$

where we have indicated with $R_{\|}^{\prime}$ the first derivative of $R_{\|}(m)$. Eqs. (40), (41) are then substituted into Eq. (38) to yield

$$
\begin{aligned}
\frac{2}{q} \frac{\partial y_{\perp}}{\partial \beta}= & R_{\|}^{2} G^{\prime}\left(y_{\|}\right) \frac{\partial^{2} y_{\perp}}{\partial m^{2}}+\left\{R_{\perp}\left[2 R_{\|} G^{\prime}\left(y_{\|}\right)+d R_{\perp} G^{\prime}\left(y_{\perp}\right)\right]+m R_{\|} R_{\|}^{\prime} G^{\prime}\left(y_{\|}\right)\right\} \frac{1}{m} \frac{\partial y_{\perp}}{\partial m} \\
& +\frac{R_{\perp}}{m}\left[R^{\prime}{ }_{\|} G\left(y_{\|}\right)+d R_{\perp}^{\prime} G\left(y_{\perp}\right)-2 m\right] .
\end{aligned}
$$

Since $R_{\|}, R_{\perp}$ are known functions of $m$ and $y_{\|}$is given by Eq. (40), the above equation is a closed PDE for $y_{\perp}$ as a function of $\beta$ and $m$. Let us observe that Eq. (36) and Eq. (27) imply that both $y_{\|}$and $y_{\perp}$ are even functions of $m$. As a consequence, the term $m^{-1} \partial y_{\perp} / \partial m$ which appears in the r.h.s. of Eq. (42) does not imply any singularity at $m=0$, since in this limit it just gives $\partial^{2} y_{\perp} / \partial m^{2}$. The PDE (42) is of course nonlinear. In particular, both $y_{\perp}$ and $\partial y_{\perp} / \partial m$ (via Eq. (40)) appear as arguments of the functions $G, G^{\prime}$. However, the dependence on $\partial^{2} y_{\perp} / \partial m^{2}$ is linear. When solving Eq. (42) numerically, one can then take advantage of this structure by resorting to some of the finite-difference algorithms especially devised for quasi-linear diffusive PDE's [20]. While these algorithms share with fully implicit methods the unconditional numerical stability, they do not require the solution of a large system of coupled non-linear equations at each integration step and are therefore easier to implement.

In order to integrate Eq. (42) we also need a set of initial and boundary conditions. The initial condition is given at $\beta=0$, i.e. in the high-temperature limit. In this limit all the correlations vanish except onsite so that, as we pointed out above, $\chi_{\perp}$ and $R_{\perp}$ coincide. Eq. (27) implies then $z_{\perp}=0$, which in turn gives via Eq. (36) the initial condition

$$
y_{\perp}(m, \beta=0)=1 \quad \text { for every } m \text {. }
$$

The magnetization per spin $m$ varies in the interval $-1 \leq m \leq 1$. However, since $y_{\perp}$ is an even function of $m$, we can restrict the integration of Eq. (42) to the interval $0 \leq m \leq 1$ provided we require $y_{\perp}$ to be indeed symmetric at $m=0$ :

$$
\frac{\partial y_{\perp}}{\partial m}(m=0, \beta)=0 \quad \text { for every } \beta \text {. }
$$

As $m$ approaches its limiting value $m=1$, the longitudinal and transverse susceptibilities $\chi_{\|}$and $\chi_{\perp}$ vanish respectively as $(1-m)^{2}$ and $1-m$. In this limit the reciprocal susceptibilities diverge like those of the non-interacting system, as the excess contribution stays 
finite. We have then again $1 / \chi_{\perp} \sim 1 / R_{\perp}$ and hence from Eqs. (27), (36)

$$
y_{\perp}(m=1, \beta)=1 \quad \text { for every } \beta \text {. }
$$

We note that for the transverse susceptibility to be positive, $y_{\perp}$ has to be positive as well. The numerical solution of Eq. (42) shows that this condition is always satisfied if the inverse temperature $\beta$ is smaller than a certain value $\beta_{c}$ such that $y_{\perp}\left(m=0, \beta_{c}\right)=0$. At $\beta=\beta_{c}$ both the transverse and (from Eq. (40)) the longitudinal susceptibilities in zero field diverge. This is the inverse critical temperature of the system. For $\beta>\beta_{c}$, we find that the condition $y_{\perp} \geq 0$ is not satisfied on the whole interval $0 \leq m \leq 1$. Instead, a certain temperature-dependent value $m_{S}(\beta)$ exists, such that $y_{\perp}$ vanishes for $m=m_{S}$ and becomes negative for $m<m_{S}$. Since from Eqs. (5), (27), (36) $y_{\perp}=0$ implies $H=0, m_{S}$ is just the spontaneous magnetization at the temperature considered. For $m<m_{S}$ not only does the transverse susceptibility behave unphysically, but the PDE itself becomes intrinsically unstable and cannot therefore be integrated numerically. Hence, we excluded the interval $\left(0, m_{S}\right)$ from the domain of integration. Below the critical temperature the boundary condition (44) is then replaced by

$$
y_{\perp}\left(m=m_{S}, \beta\right)=0 .
$$

The spontaneous magnetization $m_{S}$ is determined by checking the sign of $y_{\perp}$ as the integration with respect to $\beta$ goes on. As soon as $y_{\perp}$ becomes negative at a certain $m=m_{0}$, the spontaneous magnetization curve is widened by setting $m_{S}=m_{0}+\Delta m$, $\Delta m$ being the step of the magnetization grid.

The numerical integration of Eq. (42) has been carried out with an inverse temperature step $\Delta \beta$ initially set at $10^{-4}-10^{-5}$. This can be further reduced as the critical region is approached so as to get very close to the critical temperature, and then gradually expanded back to its initial value. The number of mesh points in the magnetization grid ranged from $\sim 10^{3}$ to $\sim 10^{4}$.

\section{Results for the simple version}

In order to assess the reliability of the approximate closure (34), we considered first the case of a linear chain of three-dimensional spins, which can be solved exactly for zero magnetic field [21]. The SCOZA predictions agree very well with the exact solution, as can be seen from Figs. 1 and 2, where the results for the susceptibility and the specific heat are shown. We recall that this one-dimensional model has no phase transition at non-zero temperature.

We then turn to the three-dimensional lattices. In this case the SCOZA does predict a phase transition at a certain temperature, which can be determined by locating the divergence of the isothermal susceptibility in zero field. The critical temperatures predicted by the SCOZA for the simple cubic (SC), body-centered cubic (BCC), and face-centered cubic (FCC) lattices are reported in Table I, together with the results of extrapolation of high-temperature expansions [10, 11] and finite-size scaling (FSS) analysis performed on 
Monte Carlo simulations [5, 6]. The agreement is very good; the error is at most $0.55 \%$. The critical temperature predicted for the SC lattice by the renormalization-group based quantum hierarchical reference theory (QHRT) in the classical limit [22] is also shown for comparison. Furthermore, the Table shows the critical internal energy per spin $u_{c}$. The SCOZA value of $u_{c}$ is actually the same for any theory where the OZ approximation (19) and the normalization condition (17) are satisfied. From Eq. (28) with $m=H=0$, $z_{\|}=z_{\perp}=1$ one has $u_{c, \mathrm{SCOZA}}=-q[P(1)-1] /[2 P(1)]$. We note that this result depends only on the type of lattice structure, so that for a certain lattice the SCOZA predicts the same $u_{c}$ for any spin dimensionality. The agreement of the SCOZA result with the data from high-temperature series expansions [9] appears to be only modest, and sensibly worse than in the Ising or $D=1$ case [1]. This is somewhat surprising, since the SCOZA is known to be exact in the $D \rightarrow \infty$ or spherical-model limit [3], so one might have expected very good agreement for intermediate $D$ as well. Since the SCOZA prediction for $u_{c}$ is not affected by the approximate closure relation (34), such a behavior seems to indicate that as the spin dimensionality is increased, the $\mathrm{OZ}$ approximation does not become more and more accurate in a monotonic fashion, but a "lowest accuracy" for some intermediate $D$ might be reached. In Fig. 3 the reduced isothermal susceptibility in zero field above the critical temperature $\chi_{\|}=\chi_{\perp}$ and the correlation length $\xi$ (BCC lattice) are compared with the results from extrapolation of high-temperature expansions [9]. In both cases the agreement appears to be very good, even in the near-critical regime when $\xi \sim 10^{2}$. As stated in the Introduction, our numerical procedure for the $D$-vector model SCOZA gives for the critical exponents the spherical model values $\gamma=2, \nu=1, \beta=1 / 2$, $\alpha=-1, \eta=0$, where the usual notation for the critical exponents has been used. In Fig. 4, the susceptibility in the critical region as a function of the reduced temperature $t=\left(T-T_{c}\right) / T_{c}$ has been plotted on a log-log scale. We have also reported the effective critical exponent $\gamma_{\text {eff }}=-d(\log \chi) / d(\log t)$, which shows that for the Heisenberg model the onset of the asymptotic critical regime as given by the SCOZA takes place for $t \simeq 10^{-3}$. This is in sharp contrast to the Ising case, also shown in the Figure, whose $\gamma_{\text {eff }}$ is affected by a very strong crossover, remaining very accurate over a quite wide range of $t$ and saturating to its inaccurate asymptotic value only for $t \simeq 10^{-5}$. The specific heat $c_{H}$ in zero field has been plotted in Fig. 5 together with a closed-form approximant [7]. Because of its mean-spherical critical behavior, the SCOZA predicts that at the critical point $c_{H}$ will saturate at a finite value, which for the FCC lattice to which the Figure refers is given by $c_{\text {peak }} / k_{\mathrm{B}}=3.03$. For the Heisenberg model $c_{H}$ is indeed finite at the critical point. The approximant reported in the figure gives $c_{\text {peak }} / k_{\mathrm{B}}=10$, but one has to take into account that this quantity depends very sensitively on the value of the critical exponent $\alpha$ used in the approximant, which in Ref. [0] was taken equal to $\alpha=-1 / 16$. As reported in the same reference, using $\alpha=-1 / 8$ shifts the peak to $c_{\text {peak }}=5.7$, which is probably a better approximation of the correct value as this choice of $\alpha$ is much closer to the best currently available estimate $\alpha \simeq-0.122$ [23]. The SCOZA predictions for the magnetic structure factor $\hat{\Gamma}(\mathbf{k})$ in zero field (BCC lattice) are shown in Fig. 6 at two different temperatures, corresponding to reduced temperatures $t \simeq 2.2 \cdot 10^{-1}$ and $t \simeq 1.4 \cdot 10^{-2}$. The agreement with the approximant [9] is very good. 
Let us now consider the results below the critical temperature. The spontaneous magnetization curve of the $\mathrm{SC}$ lattice is reported in Fig. 7, together with the results of the QHRT 22] and MC simulations [4]. In Fig. 8 the curve of the FCC lattice is compared to a Padè approximant. We see that in both cases the agreement is relatively poor, with the SCOZA results looking rather mean-field like. Actually, in the temperature range shown in the Figures, the main differences between the SCOZA and the meanfield phase boundaries can be accounted for by the shift in the critical temperature, so that they look very close to each other, provided they are plotted as a function of their respective rescaled temperatures $T / T_{c}$. The effective exponent $\beta_{\text {eff }}$ given by the SCOZA is plotted in Fig. 9. The critical behavior of the SCOZA phase boundary for the spin systems with continuous symmetry considered here is different from that found in the Ising or simple fluid models. In that case, the SCOZA critical exponents below $T_{c}$ do not coincide with the spherical ones found above $T_{c}$, and the exponent $\beta$ is given by $\beta=7 / 20=0.35$, which gives a very accurate coexistence curve. Here instead we find spherical exponents both above and below $T_{c}$. We have then $\beta=1 / 2$, as in the meanfield approximation, so that the spontaneous magnetization curve close to the critical temperature is considerably narrower than the correct one, for which $\beta \simeq 0.36$ [5, 6]. On the other hand, is seems unlikely that this mean-field like behavior alone can account for the lack of global accuracy of the SCOZA phase boundary. In fact the QHRT [22] yields for $\beta$ the non-classical value $\beta=0.41$, but nevertheless is no more accurate than SCOZA as far as overall agreement with the MC coexistence curve is concerned. An improved closure of the SCOZA equation expected to deliver more accurate results for the phase diagram is discussed in the following Section. Here we observe that, despite its modest overall accuracy, the SCOZA phase boundary becomes exact in the low-temperature limit. In this regime one can use spin-wave theory, which gives for the spontaneous magnetization [18]

$$
m_{S} \sim 1-\frac{1}{q} P(1) k_{\mathrm{B}} T+\mathcal{O}\left(T^{2}\right) .
$$

The spin-wave result for the spontaneous magnetization is compared to the SCOZA and to the mean-field predictions in Fig. 10. We see that the SCOZA predicts both the linear dependence on $T$ for $T \rightarrow 0$ and the correct slope of the $\mathcal{O}(T)$ term. Another point which deserves some attention concerns the behavior of the susceptibility across the spontaneous magnetization curve. For spin systems with continuous symmetry, we expect the longitudinal susceptibility to diverge not only at the critical point, but on the whole coexistence curve. Within the SCOZA, the coexistence curve should then merge with the spinodal. This is indeed what happens, as shown in Fig. 11, which reports the SCOZA inverse longitudinal susceptibility $1 / \chi_{\|}$along two different sub-critical isotherms. In Fig. 12 several super- and sub- critical isotherms are compared to MC simulation results [4]. The discrepancy between the SCOZA and the MC data already observed in the coexistence curve affects the sub-critical isotherms for $H \rightarrow 0$, especially for $T$ near $T_{c}$. The agreement rapidly improves as $H$ increases.

In order to assess to which extent the results discussed here depend on the ansatz (34), we also solved numerically the SCOZA PDE with a different closure relation, namely that 
of Eq. (32). We find that the thermodynamics and the phase boundary are hardly distinguishable from those already shown, the critical temperature actually getting sligthly worse: for instance, for the SC lattice one has $\beta_{c}=0.6978$, while the previous approximation (see Table I) gave $\beta_{c}=0.6968$, and the "exact" result is [6, 5, 11] $\beta_{c}=0.6930$. Moreover, our algorithm failed to find solutions below a certain sub-critical temperature. We do not know whether this is a purely numerical problem, or instead it is the sign of a deeper disease which may affect the closure (32). In this respect, we recall that in such an approximation (see Sec. II) the mean-square fluctuations of the spin in the transverse and in the longitudinal directions do not behave correctly on the coexistence curve, while the correct behavior for $m=1$ is enforced by the boundary condition (45). It is then possible that at low temperatures, as the spontaneous magnetization approaches its limiting value $m_{S}=1$, the change in behavior across the domain becomes so abrupt as to prevent the existence of a solution. Since the above closure does not introduce any improvement with respect to the approximation (34) that we have used so far, we have not investigated this point any further.

\section{Inclusion of induced spin-wave correlations}

\subsection{New closure}

We now seek to develop an improved version of the SCOZA, guided by the results for the simple version that we have just summarized. The most serious deficiency of that version is its spherical-model like coexistence curve, which considerably differs from what is expected to be the correct result. Thus we have to look for a possible leading correction that might remedy this. What we intend to do is to explicitly take into account the effect of the transverse correlations on the longitudinal susceptibility. As we will see below, this will naturally give rise to a diverging longitudinal susceptibility $\chi_{\|}$on the coexistence curve by enforcing $y_{\|}=0$ whenever $y_{\perp}=0$. On the other hand, if the effect of transverse correlations is not properly accounted for, a diverging $\chi_{\|}$at coexistence can be obtained only by heavily distorting the longitudinal part of the correlation function, with a corresponding infuence upon the internal energy and thus the SCOZA results. This is most probably what happens in the simple version that we have considered so far. For magnetization $m \rightarrow 1$ one approaches Gaussian-model behavior and these induced correlations can thus be obtained exactly by a spin-wave calculation. For general $m$, we can turn to the analysis based on $\gamma$-ordering in order to obtain their form, where $\gamma$ is the inverse range of interaction.

The induced correlations become long-ranged near the critical point and at phase coexistence. Thus their major influence will be upon the longitudinal susceptibility $\chi_{\|}$. Representing their contribution integrated over space by $\Delta$ we can write, for the longitudinal susceptibility,

$$
\chi_{\|}=\frac{R_{\|}}{y_{\|}}=\frac{R_{\|}}{y_{l}}+\Delta
$$


where the new quantity $y_{l}$ represents the contribution of the MSA-like part of the correlation function. Solving with respect to $y_{l}$ one then has

$$
y_{l}=\frac{y_{\|} R_{\|}}{R_{\|}-y_{\|} \Delta},
$$

and there will be a quantity $z_{l}$ related to $y_{l}$ by Eq. (36). The direct contribution these induced correlations will make to the internal energy will be minor and less crucial according to $\gamma$-ordering, so we neglect it here for simplicity. The quantity $y_{l}$ is then used instead of $y_{\|}$in Eq. (38) to evaluate the longitudinal part of the internal energy. This requires having an expression for $\Delta$. The analysis is most conveniently given in the diagrammatic language developed [24] to describe the $\gamma$-ordering used by Høye and Stell. In that language, the correlation functions are built up of chain bonds (representing chains of pair interactions) and hypervertices (representing the short-range components of the correlation functions). The leading contribution beyond the MSA to the pair correlation function $\Gamma(\mathbf{r})$ of a fluid system has the diagrammatic form

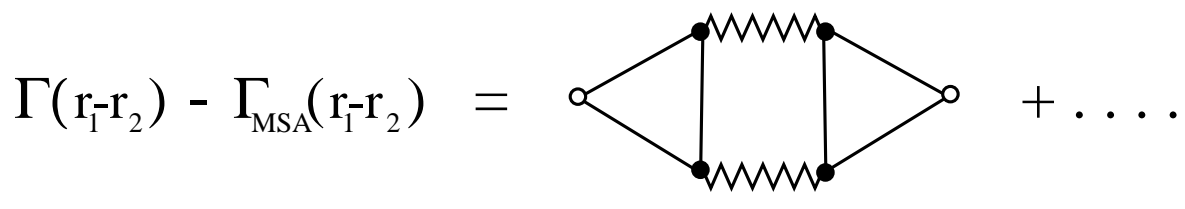

where the triangles represent the three-body correlation function of the reference system with purely short-range interactions (including self-correlations where two or all three of the particles can be identical), the white circles (or "root points") represent the coordinates $\mathbf{r}_{1}, \mathbf{r}_{2}$, and the full circles represent coordinates that are integrated over. The zigzag chain bond $C(\mathbf{r})$ (not to be confused with the direct correlation function $c(\mathbf{r})$ ) is defined by

$\mathrm{C}\left(\mathrm{r}_{1}-\mathrm{r}_{2}\right)=$ oMMno $=0 \ldots+0 \ldots+\cdots$

where the solid and dashed lines represent respectively the two-body correlation function of the reference system $\Gamma_{R}(\mathbf{r})$ and the perturbing function $-\beta \varphi(\mathbf{r})$. In Fourier space one then has

$$
\hat{C}(\mathbf{k})=\frac{-\beta \hat{\varphi}(\mathbf{k})}{1+\beta \hat{\varphi}(\mathbf{k}) \hat{\Gamma}_{\mathrm{R}}(\mathbf{k})} .
$$

The above result can be generalized to spin systems by utilizing Onsager's recipe for regarding different orientations as different species of a mixture 25. In particular, the reference system consists of non-interacting spins, and is mapped into a multicomponent, but simple, hard-core lattice gas whose $n$-body correlation functions $\Gamma_{\mathrm{R}}^{(n)}\left(\mathbf{r}_{1} \ldots \mathbf{r}_{n}\right)$ are 
non-vanishing only when the coordinates $\mathbf{r}_{1} \ldots \mathbf{r}_{n}$ coincide. In particular, the three-body hypervertex has the form

$$
\Gamma_{\mathrm{R}}^{(3)}\left(\mathbf{r}_{1}, \mathbf{r}_{2}, \mathbf{r}_{3}\right)=\mathbf{b} \delta_{\mathbf{r}_{1} \mathbf{r}_{2}} \delta_{\mathbf{r}_{2} \mathbf{r}_{3}}
$$

In the multicomponent case, the $\Gamma_{\mathrm{R}}^{(3)}$ as well as the chain bond should be summed over the various species. One finds

$$
\sum_{s} s \frac{\partial}{\partial \mu_{s}}=\frac{\partial}{\partial H}
$$

where $\mu_{s}$, the chemical potential has the form $s H+$ const. Thus the sum on the lefthand side can be replaced by a differentiation with respect to $H$, which implies that the resulting $\boldsymbol{\Gamma}_{\mathrm{R}}^{(3)}$ can again be represented by the one-component form Eq. (51). For the multicomponent system considered here, $\mathbf{b}$ is a three-index tensor with components $b_{i j k}$, where each index can assume $D$ values corresponding to the longitudinal and each of the $D-1=d$ transverse components of the spin. $b_{i j k}$ is determined by the sum rule

$$
b_{i j k}=\frac{1}{N} \sum_{\mathbf{r}_{1}, \mathbf{r}_{2}, \mathbf{r}_{3}} \Gamma_{\mathrm{R}}^{(3) i j k}\left(\mathbf{r}_{1}, \mathbf{r}_{2}, \mathbf{r}_{3}\right)=-\left.\frac{\partial^{3} \beta \omega_{\mathrm{R}}}{\partial \beta H_{i} \partial \beta H_{j} \partial \beta H_{k}}\right|_{H_{\|}=H, H_{\perp}=0},
$$

where $N$ is the number of lattice sites and $\omega_{\mathrm{R}}$ is the grand potential per site of the reference system. This is the analog of the compressibility rule (12), (13) for the twobody correlation function. We recall that for the reference system the latter gives $\chi_{\|}=R_{\|}$, $\chi_{\perp}=R_{\perp}$, where the quantities $R_{\|}, R_{\perp}$ have been defined in Eqs. (15), (16). According to Eq. (12), the contribution $\Delta$ in Eq. (48) is obtained by integrating over space the leading contribution to $\boldsymbol{\Gamma}(\mathbf{r})-\boldsymbol{\Gamma}_{\mathrm{MSA}}(\mathbf{r})$ shown above, where each of the white circles corresponds to a longitudinal root point, and the full circles involve both a spatial integration and a summation over the component index. We observe that the matrix of the two-body correlation function $\Gamma_{\mathrm{R}}^{i j}$ (see Eqs. (9), (10)) and that of the nearest-neighbor spin-spin interaction $s_{\mathbf{r}^{i}}^{i} s_{\mathbf{r}^{\prime}}^{j}$ are diagonal. Eq. (50) then implies that this is true also for the matrix of the chain bond $C_{i j}(\mathbf{r})$, i.e., the zigzag bond is non-vanishing only if its endpoints correspond to the same spin component. It is then readily seen that in performing the summation over the indexes, the only two terms which contribute to $\Delta$ are those in which both the three-body hypervertices are either of the type $b_{\|\|\|\|}$or of the type $b_{\| \perp \perp}$. By setting $b_{\| \perp \perp}=b$ and applying Eq. (53) we find

$$
b=\frac{\partial R_{\perp}}{\partial \beta H}=R_{\|} R_{\perp}^{\prime}=\frac{R_{\perp}}{m}\left(R_{\|}-R_{\perp}\right),
$$

where the prime denotes differentiation with respect to $m$, and Eq. (6) has been used. We note that, since $R_{\|}-R_{\perp}=\mathcal{O}\left(m^{2}\right)$ for $m \rightarrow 0, b$ vanishes for $m=0$. In the limit $m \rightarrow 1, b_{\|\|\|\|}=R_{\|} R_{\|}^{\prime}$ can be disregarded with respect to $b$ as then one has $b \sim(1-m)^{2}$, $b_{\|\|\|\|} \sim(1-m)^{3}$ (see the following section). We have then

$$
\Delta=\frac{1}{2} d b^{2} B
$$


where the factor $1 / 2$ is the graph symmetry factor with respect to the interchange of the two chain bonds, $d$ is the number of the transverse components, and $B$ is given by

$$
B=\sum_{\mathbf{r}} C_{\perp}^{2}(\mathbf{r})
$$

$C_{\perp}(\mathbf{r})$ being a short-hand notation for $C_{\perp \perp}(\mathbf{r})$. The chain bond follows from Eq. (50), or equivalently from the MSA-like form of the correlation function, whose Fourier transform is given by Eqs. (18), (20). The transverse part is then

$$
\hat{\Gamma}_{\perp}(\mathbf{k})=\frac{R_{\perp}}{1-R_{\perp}\left(c_{0}^{\perp}+q c_{1}^{\perp} \gamma(\mathbf{k})\right)},
$$

where $c_{0}^{\perp}$ can be regarded as representing the perturbing interaction inside the hard core. With $c_{1}^{\perp}=\beta$ as in the MSA, $R_{\perp}$ is clearly the two-body hypervertex of the reference system. With $c_{1}^{\perp} \neq \beta$, this interpretation can be kept as the difference $c_{1}^{\perp}-\beta$ is small and will become even smaller when including the new contribution $\Delta$. We then write

$$
\hat{\Gamma}_{\perp}(\mathbf{k})=R_{\perp}+R_{\perp}^{2} \hat{C}_{\perp}(\mathbf{k})
$$

Along with Eq. (57) this yields for the chain bond in Fourier space

$$
\hat{C}_{\perp}(\mathbf{k})=\frac{z_{\perp}}{R_{\perp} P\left(z_{\perp}\right)}\left[\frac{\gamma(\mathbf{k})}{1-z_{\perp} \gamma(\mathbf{k})}-\frac{P\left(z_{\perp}\right)-1}{z_{\perp}}\right] .
$$

Note that since $\Gamma_{\perp}(r=0)=R_{\perp}$ we have $C_{\perp}(r=0)=0$, which also follows from Eq. (59) using the definition (23) for the integral $P(z)$. Now the integral of the double chain bond can be evaluated in a straightforward way by Fourier methods:

$$
B=\int \frac{d^{3} \mathbf{k}}{(2 \pi)^{3}} \hat{C}_{\perp}^{2}(\mathbf{k})=\left(\frac{1}{R_{\perp} P\left(z_{\perp}\right)}\right)^{2}\left[z_{\perp} P^{\prime}\left(z_{\perp}\right)-P\left(z_{\perp}\right)\left(P\left(z_{\perp}\right)-1\right)\right],
$$

where we have set $P^{\prime}(z)=d P(z) / d z$. Inserting Eqs. (54) and (60) into Eq. (55) yields the sought quantity $\Delta$ to be used in Eq. (49).

\subsection{Spin-wave limit}

As $m \rightarrow 1$ the transverse modes represent harmonic oscillators that can be solved exactly by Gaussian integrals. With the added contribution $\Delta$ of Eq. (48) the SCOZA will recover the exact spin-wave solution also for the longitudinal susceptibility as $m \rightarrow 1$. I.e. thermodynamic self-consistency is immediately fulfilled with $c_{1}^{\|}=c_{1}^{\perp}=\beta$ in this limiting case also at phase equilibrium where $P^{\prime}\left(z_{\perp}\right) \rightarrow \infty$.

To show that SCOZA has this behavior, we first evaluate $R_{\perp}$ as $m \rightarrow 1$. By setting $\nu=d / 2$ we have

$$
m=\left\langle s_{\mathbf{r}}^{\|}\right\rangle=\left\langle\sqrt{1-d\left(s_{\mathbf{r}}^{\perp}\right)^{2}}\right\rangle \sim 1-\nu\left\langle\left(s_{\mathbf{r}}^{\perp}\right)^{2}\right\rangle=1-\nu R_{\perp}
$$


so that in this limit

$$
R_{\perp}=\frac{1-m}{\nu}
$$

If we set $c_{1}^{\perp}=\beta$, Eq. (25) then gives

$$
q \beta(1-m)=\nu z_{\perp} P\left(z_{\perp}\right) .
$$

We also note that in the same limit $R_{\|}=\left\langle\left(s_{\mathbf{r}}^{\|}-\left\langle s_{\mathbf{r}}^{\|}\right\rangle\right)^{2}\right\rangle=(1-m)^{2} / \nu$, so that it can be neglected compared with $R_{\perp}$. Eqs. (54) and (62) then imply

$$
b=-\frac{(1-m)^{2}}{\nu^{2}} .
$$

This together with Eqs. (55), (60), (63) yields

$$
\Delta=(1-m)^{2} \frac{1}{\nu P^{2}\left(z_{\perp}\right)}\left[z_{\perp} P^{\prime}\left(z_{\perp}\right)-P\left(z_{\perp}\right)\left(P\left(z_{\perp}\right)-1\right)\right] .
$$

In order to obtain the longitudinal susceptibility from Eq. (48) one also needs knowledge of $y_{l}$. Eq. (25) with $c_{1}^{\|}=\beta$ and $z=z_{l}$ implies $z_{l} \rightarrow q \beta R_{\|} \rightarrow q \beta(1-m)^{2} / \nu$ (i.e. $P\left(z_{l}\right) \rightarrow 1$ ), so that Eq. (36) gives

$$
y_{l}=1-q \beta R_{\|} \rightarrow 1 \text {. }
$$

Substituting Eqs. (65) and (66) into Eq. (48) we obtain

$$
\chi_{\|}=\frac{1}{\nu}(1-m)^{2} \frac{z_{\perp} P^{\prime}\left(z_{\perp}\right)+P\left(z_{\perp}\right)}{P^{2}\left(z_{\perp}\right)} .
$$

This can be compared with the result obtained by differentiating the transverse susceptibility with respect to $m$. For $m \rightarrow 1$ only the transverse correlations contribute to the internal energy, and the SCOZA equation (31) reduces to

$$
\frac{1}{q} \frac{\partial}{\partial \beta}\left(\frac{1}{\chi_{\perp}}\right)=-1-\frac{\partial}{\partial m}\left(\nu R_{\perp} \frac{P\left(z_{\perp}\right)-1}{z_{\perp} P\left(z_{\perp}\right)}\right)=-1-\frac{\nu}{q \beta} P^{\prime}\left(z_{\perp}\right) \frac{\partial z_{\perp}}{\partial m},
$$

where again Eq. (25) with $c_{1}^{\perp}=\beta$ has been used. In the same limit we then also have with Eqs. (25) and (36)

$$
y_{\perp} \rightarrow P\left(z_{\perp}\right)-q \beta R_{\perp}
$$

so that from Eqs. (27) and (36)

$$
\frac{1}{q} \frac{\partial}{\partial \beta}\left(\frac{1}{\chi_{\perp}}\right)=-1+\frac{\nu}{q(1-m)} P^{\prime}\left(z_{\perp}\right) \frac{\partial z_{\perp}}{\partial \beta} .
$$

The partial derivatives of $z_{\perp}$ which appear in Eqs. (68), (70) are obtained by differentiating Eq. (63) with respect to $\beta$ and $m$. This gives

$$
\begin{aligned}
\frac{\partial z_{\perp}}{\partial \beta} & =\frac{q(1-m)}{\nu\left[z_{\perp} P^{\prime}\left(z_{\perp}\right)+P\left(z_{\perp}\right)\right]}, \\
\frac{\partial z_{\perp}}{\partial m} & =-\frac{q \beta}{\nu\left[z_{\perp} P^{\prime}\left(z_{\perp}\right)+P\left(z_{\perp}\right)\right]} .
\end{aligned}
$$


By substituting Eq. (71) into Eq. (70) it is readily seen that the consistency condition Eq. (68) is satisfied. Finally by using Eqs. (61), (27), (36), (69), (72) the longitudinal susceptibility becomes

$$
\frac{1}{\chi_{\|}}=\frac{\partial}{\partial m}\left(m \frac{y_{\perp}}{R_{\perp}}\right) \rightarrow \nu \frac{\partial}{\partial m}\left(\frac{P\left(z_{\perp}\right)}{1-m}\right) \rightarrow \frac{\nu}{(1-m)^{2}} \frac{P^{2}\left(z_{\perp}\right)}{z_{\perp} P^{\prime}\left(z_{\perp}\right)+P\left(z_{\perp}\right)},
$$

in agreement with Eq. (67). Thus the contribution $\Delta$ in Eq. (48) fully accounts for the spin waves in the limit $m \rightarrow 1$, and SCOZA yields full thermodynamic self-consistency with $c_{1}^{\|}=c_{1}^{\perp}=\beta$ in this limit. Without this term Eq. (67) reduces to $\chi_{\|}=(1-m)^{2} / \nu=R_{\|}$as a consequence of the assumption $c_{1}^{\|}=\beta$ that leads to Eq. (65). However, SCOZA, that does not "know" about this assumption, still requires Eq. (73) to be fulfilled (with $c_{1}^{\perp} \simeq \beta$ ) on the basis of the MSA-type expression (18), which implies $\chi_{\|}=R_{\|} /\left[1-R_{\|}\left(c_{0}^{\|}+q c_{1}^{\|}\right)\right]$. At phase equilibrium where $\chi_{\|} \rightarrow \infty$ since $P^{\prime}\left(z_{\perp}\right) \rightarrow \infty$ this implies that $c_{0}^{\|}+c_{1}^{\|} \rightarrow \infty$ as $R_{\|} \rightarrow 0$, so that $c_{1}^{\|}-\beta$ is no longer small and the longitudinal part of the correlation function will be heavily distorted.

\section{Conclusions}

In this paper a simple version of our SCOZA is studied, in which the parallel and transverse components of the onsite spin-spin correlation $R$ are assumed to be those of the hightemperature susceptibility. This is found to be quantitatively accurate in its assessment of $T_{c}$ but not in several other respects. This treatment neglects the effects of the transverse correlations on the longitudinal susceptibility. We then develop a version of SCOZA in which the neglected correlations are included to lowest order in their $\gamma$-ordered expansion. This guarantees that spin waves are well-accounted for in a way that becomes exact in the $m \rightarrow 1$ limit. The quantitative assessment of the resulting version of SCOZA will require a significant computational effort and is not attempted here. We defer it to our next paper on the $D$-dimensional spin model.

\section{Acknowledgments}

D.P. gratefully acknowledges the support of the Division of Chemical Sciences, Office of Basic Energy Sciences, Office of Energy Research, U.S. Department of Energy for his work at Stony Brook. G.S. gratefully acknowledges the support of the National Science Foundation. 


\section{References}

[1] R. Dickman and G. Stell, Phys. Rev. Lett. 77, 996 (1996). D. Pini, G. Stell, and R. Dickman, Phys. Rev. E 57, 2862 (1998).

[2] D. Pini, G. Stell, and J. S. Høye, Int. J. Thermophys. 19, 1029 (1998). D. Pini, G. Stell, and N. B. Wilding, Mol. Phys. 95, 483 (1998).

[3] J. S. Høye and G. Stell, Physica A 244, 176 (1997).

[4] K. Binder and H. Müller-Krumbhaar, Phys. Rev. B 7, 3297 (1973).

[5] C. Holm and W. Janke, Phys. Rev. B 48, 936 (1993).

[6] K. Chen, A. M. Ferrenberg, and D. P. Landau, Phys. Rev. B 48, 3249 (1993).

[7] C. Domb and R. G. Bowers, J. Phys. C 2, 755 (1969).

[8] R. L. Stephenson and P. J. Wood, J. Phys. C 3, 90 (1970).

[9] D. S. Ritchie and M. E. Fisher, Phys. Rev. B 5, 2668 (1972).

[10] S. McKenzie, C. Domb, and D. L. Hunter, J. Phys. A 15, 3899 (1982).

[11] P. Butera and M. Comi, Phys. Rev. B 52, 6185 (1995).

[12] H. Li and T. Chen, Z. Phys. B 100, 283 (1996).

[13] Y. Song and X. Gou, Phys. Lett. A 245, 479 (1998).

[14] J. S. Høye, D. Pini, and G. Stell, Physica A 279, 213 (2000).

[15] See, for instance, J. P. Hansen and I. R. McDonald, Theory of Simple Liquids, Academic Press, London, 1986.

[16] S. Katsura, T. Morita, S. Inawashiro, T. Horiguchi, and Y. Abe J. Math. Phys. 12, 892 (1971); 12, 895 (1971); 12, 981 (1971); 12, 986 (1971).

[17] G.S. Joyce, J. Math. Phys. 12, 1390 (1971); J. Phys. C4, L53 (1971); J. Phys. A5, L65 (1972).

[18] R. E. Watson, M. Blume, and G. H. Vineyard, Phys. Rev 181, 811 (1969).

[19] See, for instance, S. K. Ma, Statistical Mechanics, World Scientific, Singapore, 1985.

[20] W.F. Ames, Numerical Methods for Partial Differential Equations (Academic, New York, 1977).

[21] M. E. Fisher, Am. J. Phys. 32, 343 (1964). H. E. Stanley, Phys. Rev. 179, 570 (1969). 
[22] P. Gianinetti and A. Parola, Phys. Rev. B 63, 104414 (2001), and private communication.

[23] R. Guida and J. Zinn-Justin, J. Phys. A 31, 8103 (1998).

[24] P. C. Hemmer, J. Math. Phys. 5, 75 (1964). J. L. Lebowitz, G. Stell, and S. Baer, J. Math. Phys. 6, 1282 (1965). J. S. Høye and G. Stell, J. Chem. Phys. 61, 562 (1974). J. S. Høye, in Proceedings NATO Advanced Study Institute on New Approaches to Problems in Liquid State Theory, eds. C. Caccamo, J.-P. Hansen, and G. Stell (Kluwer Academic Publishers, Dordrecht, 1999).

[25] L. Onsager, Ann. N. Y. Acad. Sci. 51, 627 (1949).

TABLES

\begin{tabular}{cccccc}
\hline \hline lattice & $\beta_{c, \mathrm{SCOZA}}$ & $\beta_{c, \mathrm{QHRT}}$ & $\beta_{c, \mathrm{ex}}$ & $u_{c, \mathrm{SCOZA}}$ & $u_{c, \mathrm{ex}}$ \\
\hline $\mathrm{SC}$ & 0.6968 & 0.7047 & $0.6930^{a}$ & -1.0216 & $-0.987 \pm 6^{c}$ \\
$\mathrm{BCC}$ & 0.4887 & & $0.4868^{a}$ & -1.1289 & $-1.092 \pm 8^{c}$ \\
$\mathrm{FCC}$ & 0.3160 & & $0.3148^{b}$ & -1.5379 & $-1.4808 \pm 30^{c}$ \\
\hline \hline
\end{tabular}

Critical parameters of the classical Heisenberg model on a SC, BCC, and FCC lattice. The inverse critical temperature $\beta_{c}$ and the critical internal energy per spin $u_{c}$ predicted by the SCOZA are compared with the "exact" $\beta_{c, \text { ex }}, u_{c, \text { ex }}$ determined by extrapolation of high-temperature expansions and by MC simulations. Energies and temperatures are in units of the interaction strength $J$ and of $J / k_{\mathrm{B}}$ respectively. $a$ : results from hightemperature expansions [11] and MC-FSS simulations [5, 6]. $b$ : from high-temperature expansions [10]. $c$ : from high-temperature expansions [9]. For the SC lattice, the inverse critical temperature predicted by the quantum hierarchical reference theory (QHRT) [22] in the classical limit is also reported. 


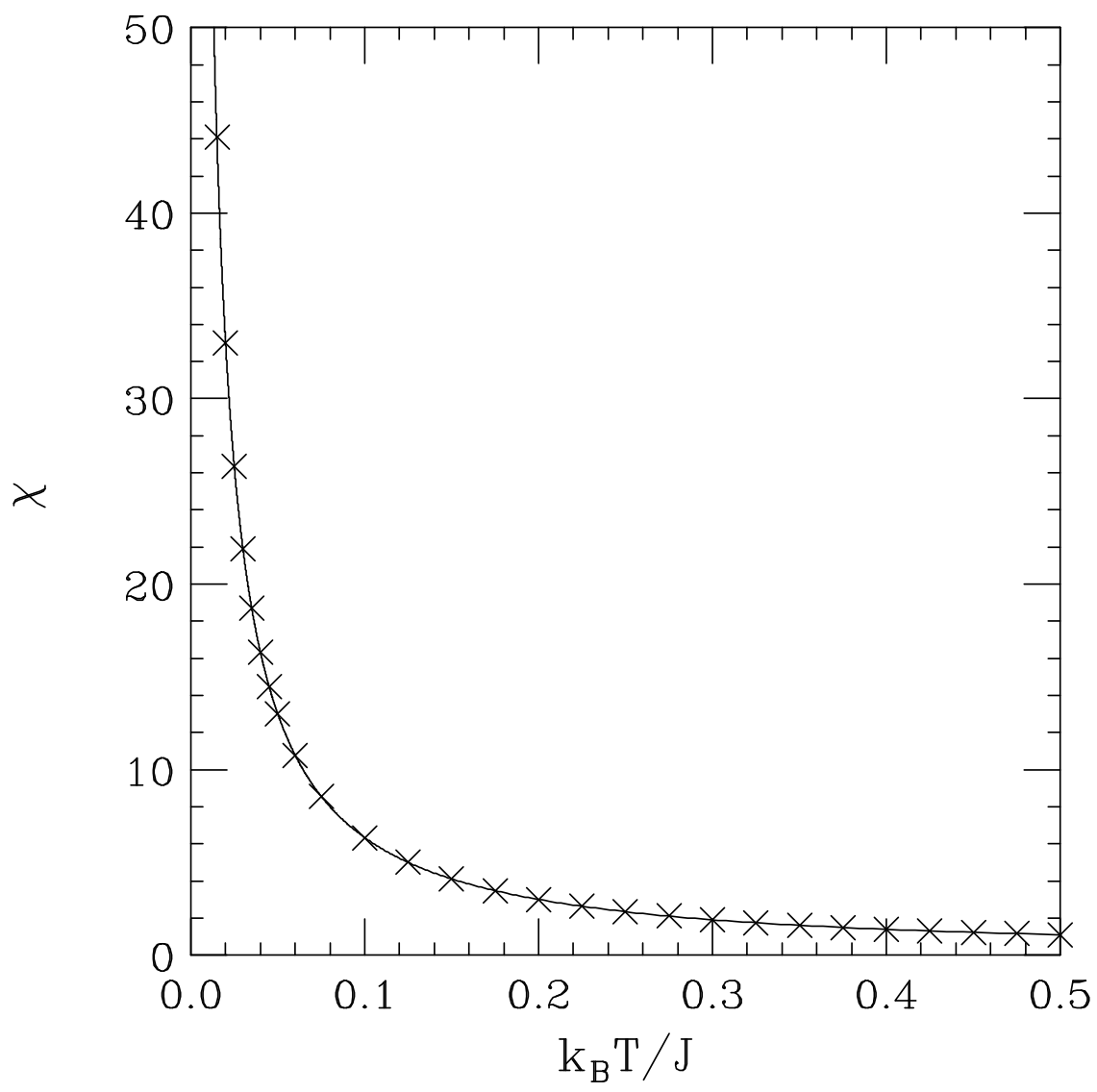

Figure 1: Reduced isothermal susceptibility $\chi$ in zero field of the classical Heisenberg model on a one-dimensional chain as a function of the dimensionless temperature $k_{\mathrm{B}} T / J$, $J$ being the spin-spin coupling strength. Solid line: SCOZA. Crosses: exact result [21]. 


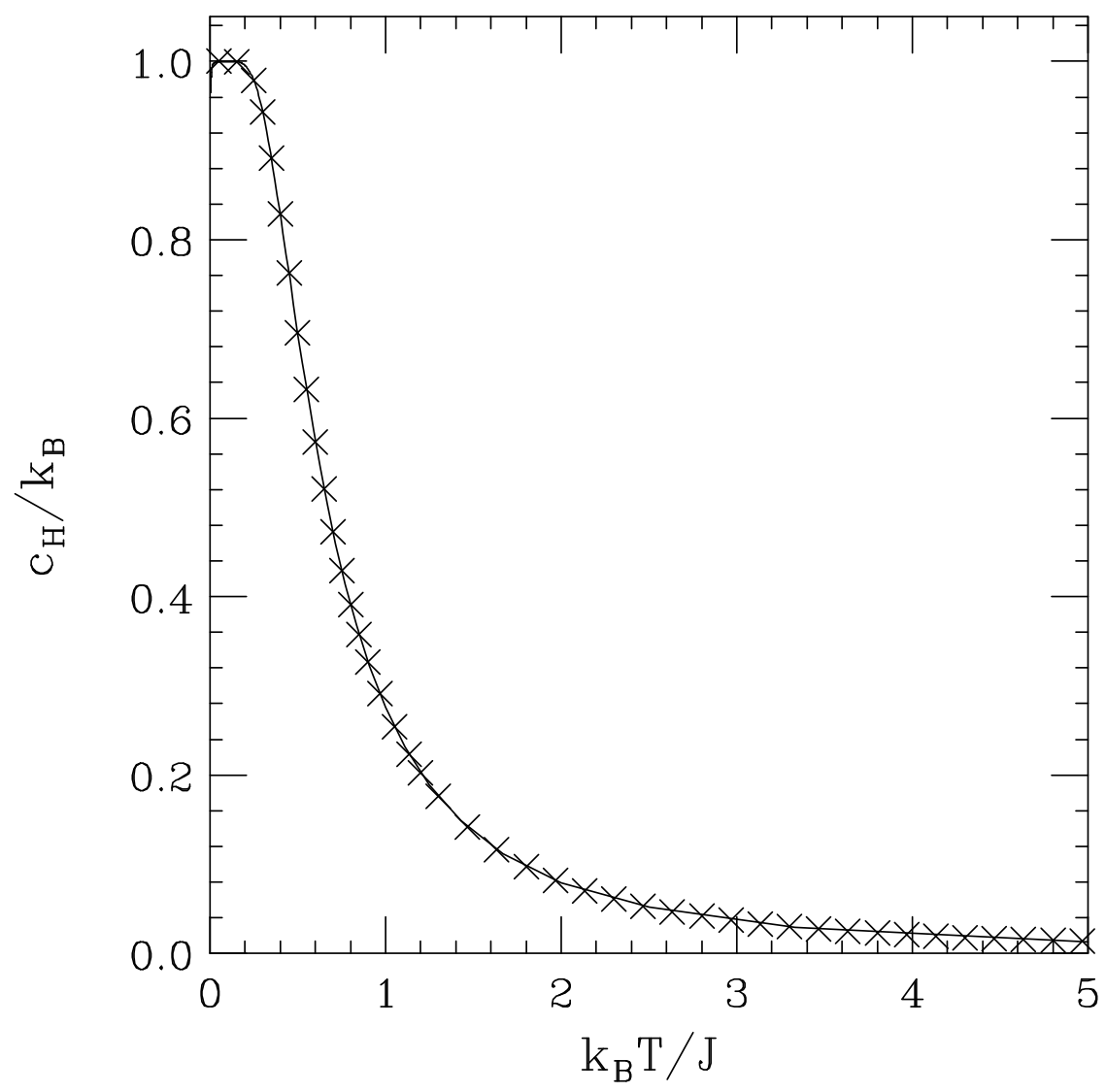

Figure 2: Specific heat per spin $c_{H}$ in zero field of the classical Heisenberg model on a one-dimensional chain as a function of the dimensionless temperature $k_{\mathrm{B}} T / J$. Solid line: SCOZA. Crosses: exact result [21]. 


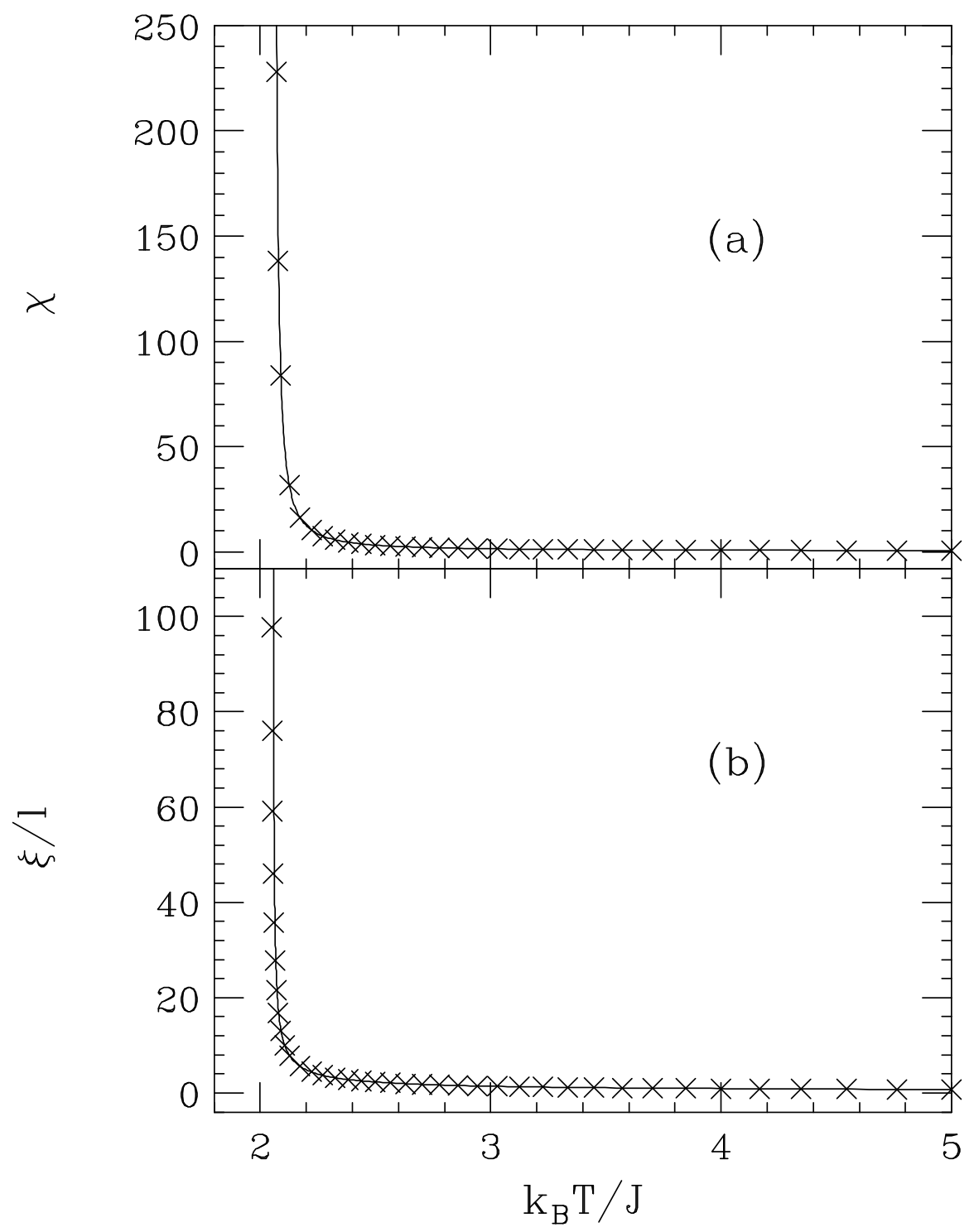

Figure 3: Reduced isothermal susceptibility $\chi$ (a) and correlation length $\xi$ (b) of the classical Heisenberg model in zero field above the critical temperature (BCC lattice). $\xi$ is in units of the lattice spacing $l$. Crosses: SCOZA. Solid line: approximant [9]. 




Figure 4: Reduced isothermal susceptibility $\chi$ in zero field (a) and effective critical exponent $\gamma_{\text {eff }}$ (b) of the classical Heisenberg model (BCC lattice) as a function of the reduced temperature $\left(T-T_{c}\right) / T_{c}$. Solid line: SCOZA. Dashed line: approximant [9]. Dotted line in panel (b): SCOZA result for the Ising model [1]. 


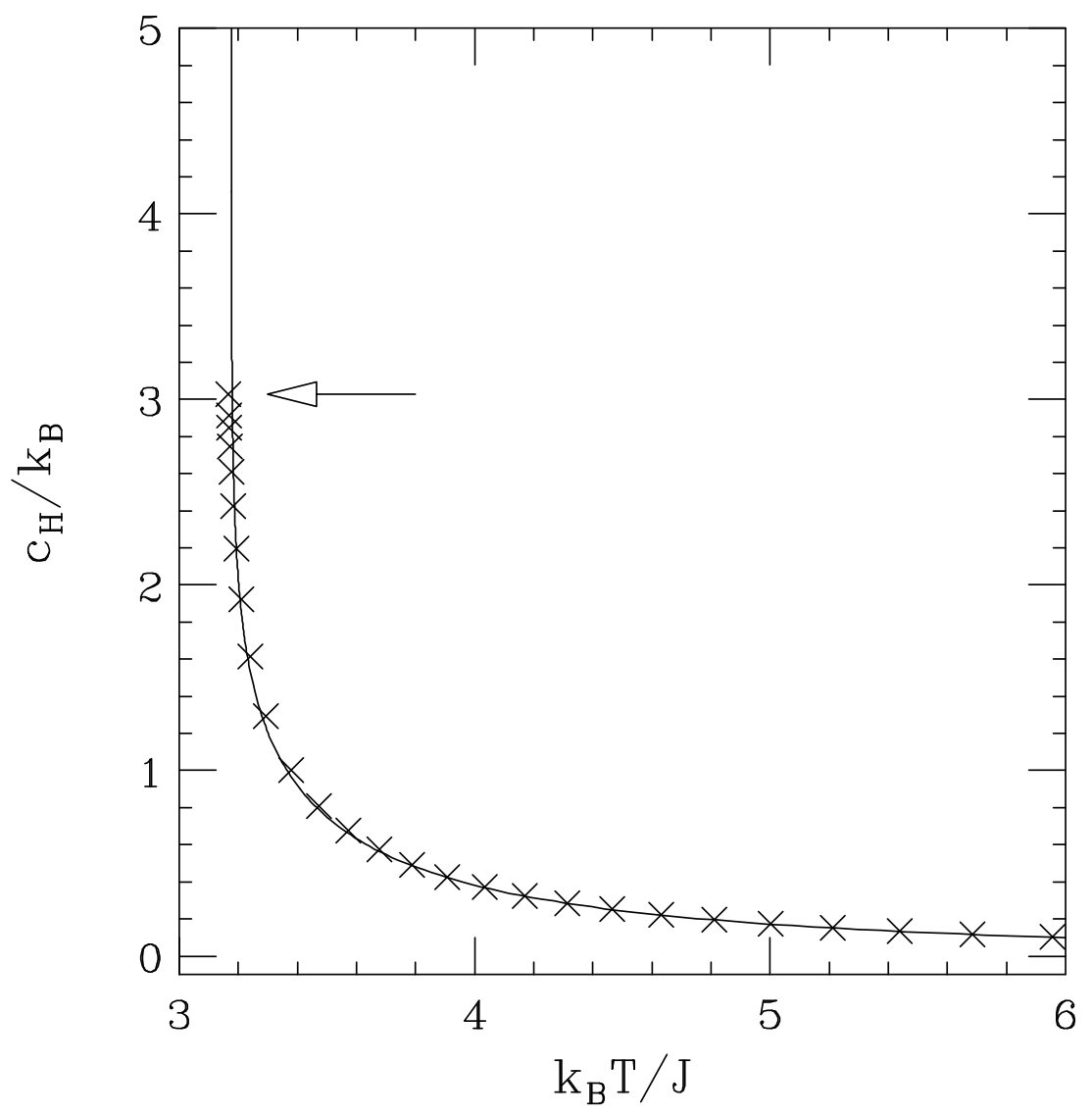

Figure 5: Specific heat per spin $c_{H}$ in zero field of the classical Heisenberg model above the critical temperature (FCC lattice). Crosses: SCOZA. Solid line: approximant [7]. The arrow marks the height of the peak of the SCOZA specific heat $c_{\text {peak }} \simeq 3.03$. 


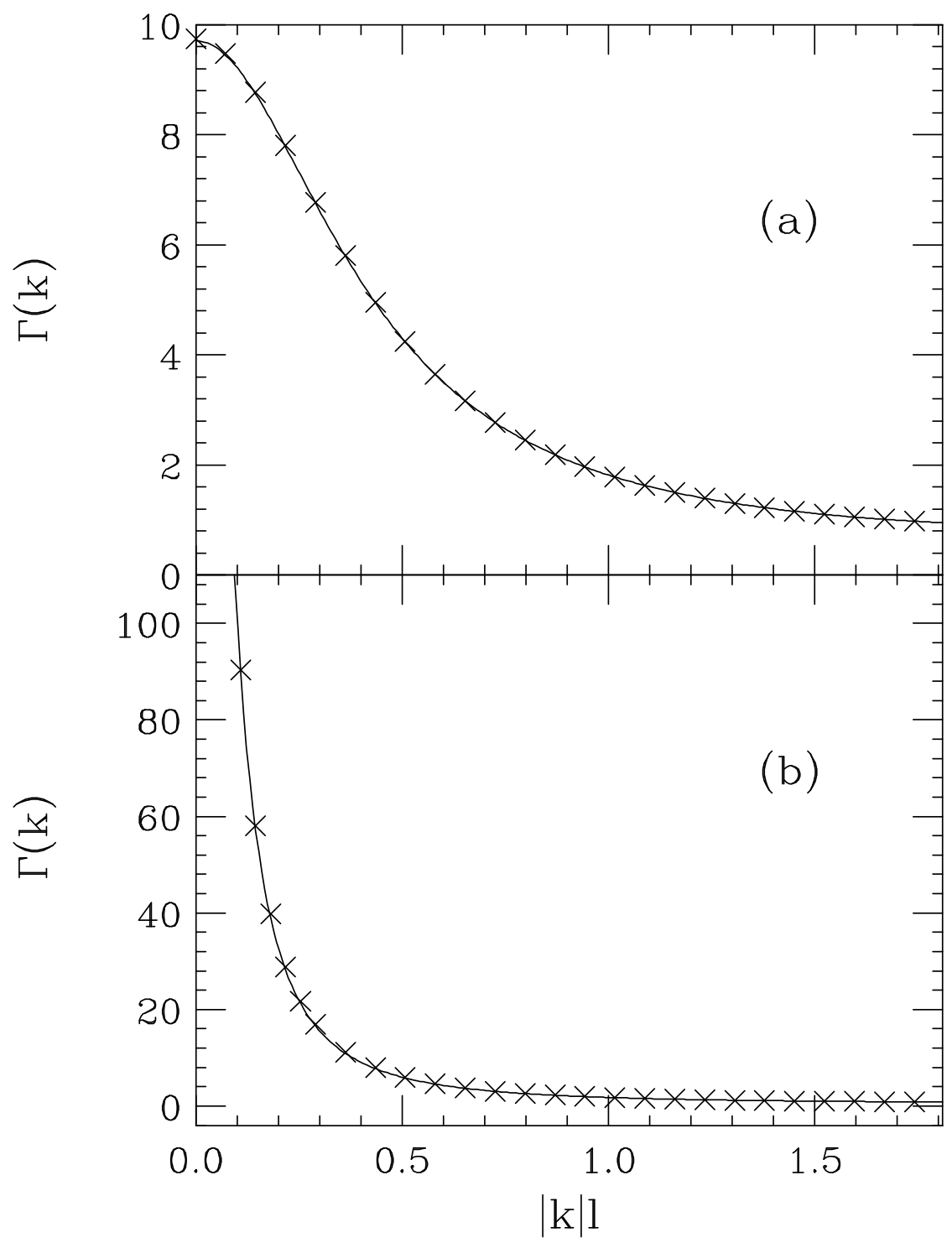

Figure 6: Magnetic structure factor $\Gamma(\mathbf{k})$ in zero field of the classical Heisenberg model on a BCC lattice along the direction $k_{x}=k_{y}=k_{z}$. The norm of the wavevector $|\mathbf{k}|$ is in units of the reciprocal of the lattice spacing $l$. The results shown are for inverse temperature $\beta=0.4$ (a) and $\beta=0.48$ (b). Crosses: SCOZA. Solid line: approximant [9]. 


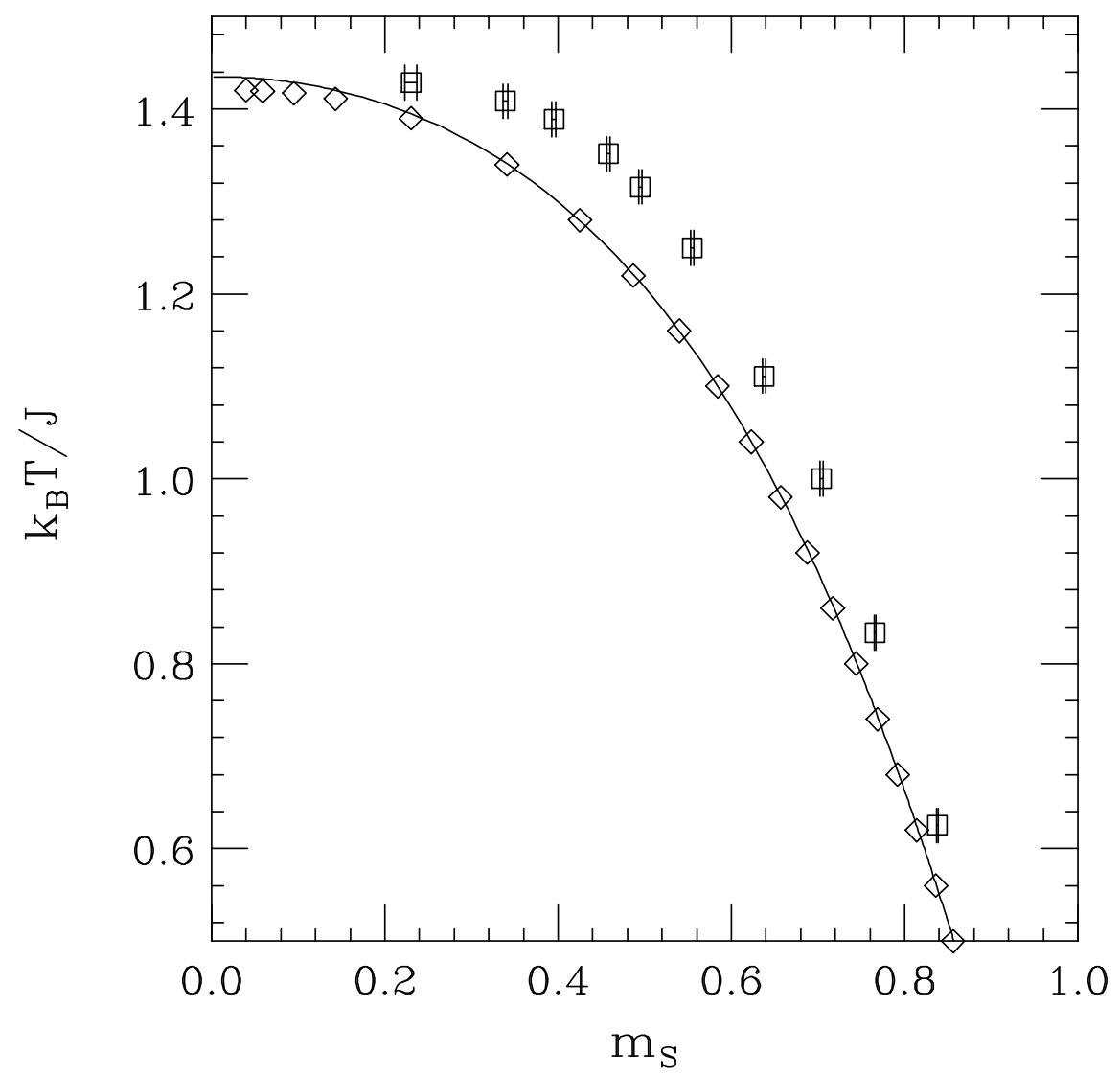

Figure 7: Spontaneous magnetization curve of the classical Heisenberg model on a SC lattice. Solid line: SCOZA. Diamonds: QHRT [22]. Squares: MC simulation results [4]. 


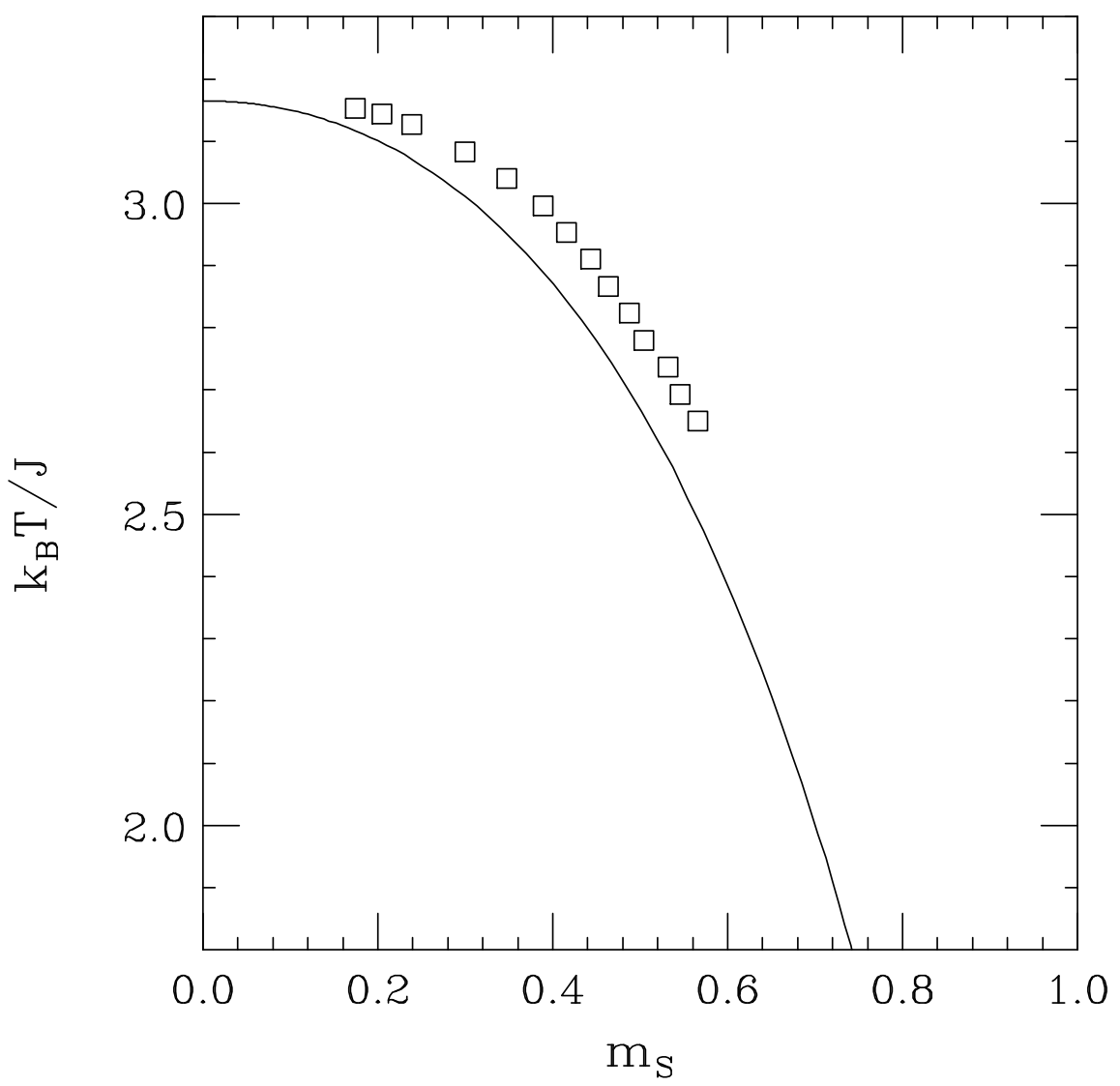

Figure 8: Spontaneous magnetization curve of the classical Heisenberg model on a FCC lattice. Solid line: SCOZA. Squares: Padè approximant [8]. 




Figure 9: SCOZA effective exponent $\beta_{\text {eff }}$ for the curvature of the spontaneous magnetization curve of the classical Heisenberg model (SC lattice) as a function of the reduced temperature $\left(T_{c}-T\right) / T_{c}$. 


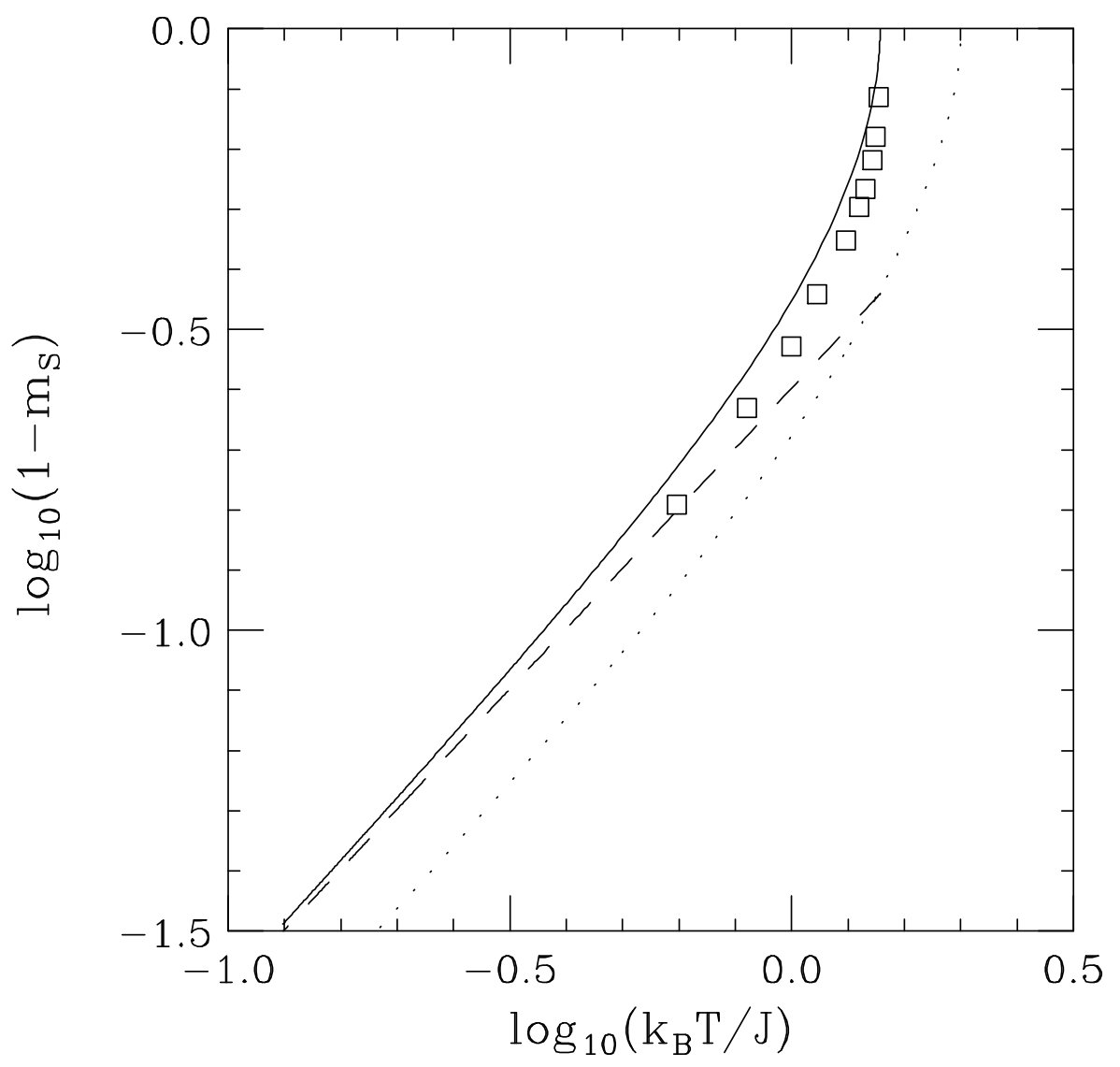

Figure 10: Low-temperature behavior of the spontaneous magnetization curve of the classical Heisenberg model (SC lattice). Solid line: SCOZA. Dotted line: mean-field theory. Squares: MC simulation data [4]. Dashed line: spin-wave theory result to $\mathcal{O}(T)$ [18]. 


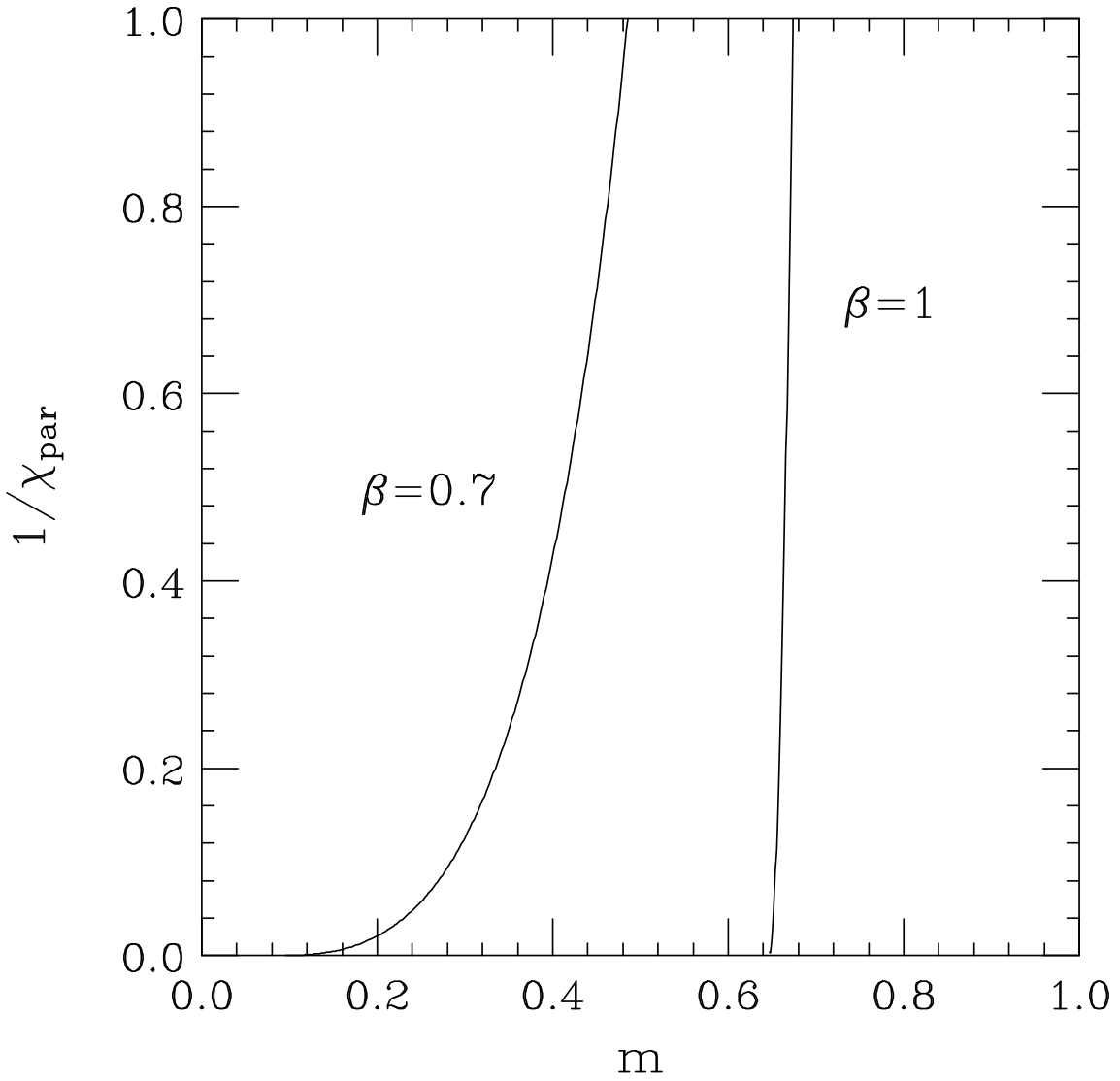

Figure 11: Reciprocal of the reduced longitudinal susceptibility $1 / \chi_{\|}$of the classical Heisenberg model (SC lattice) outside the phase boundary. The SCOZA results are shown for two different values of the inverse temperature $\beta$. 


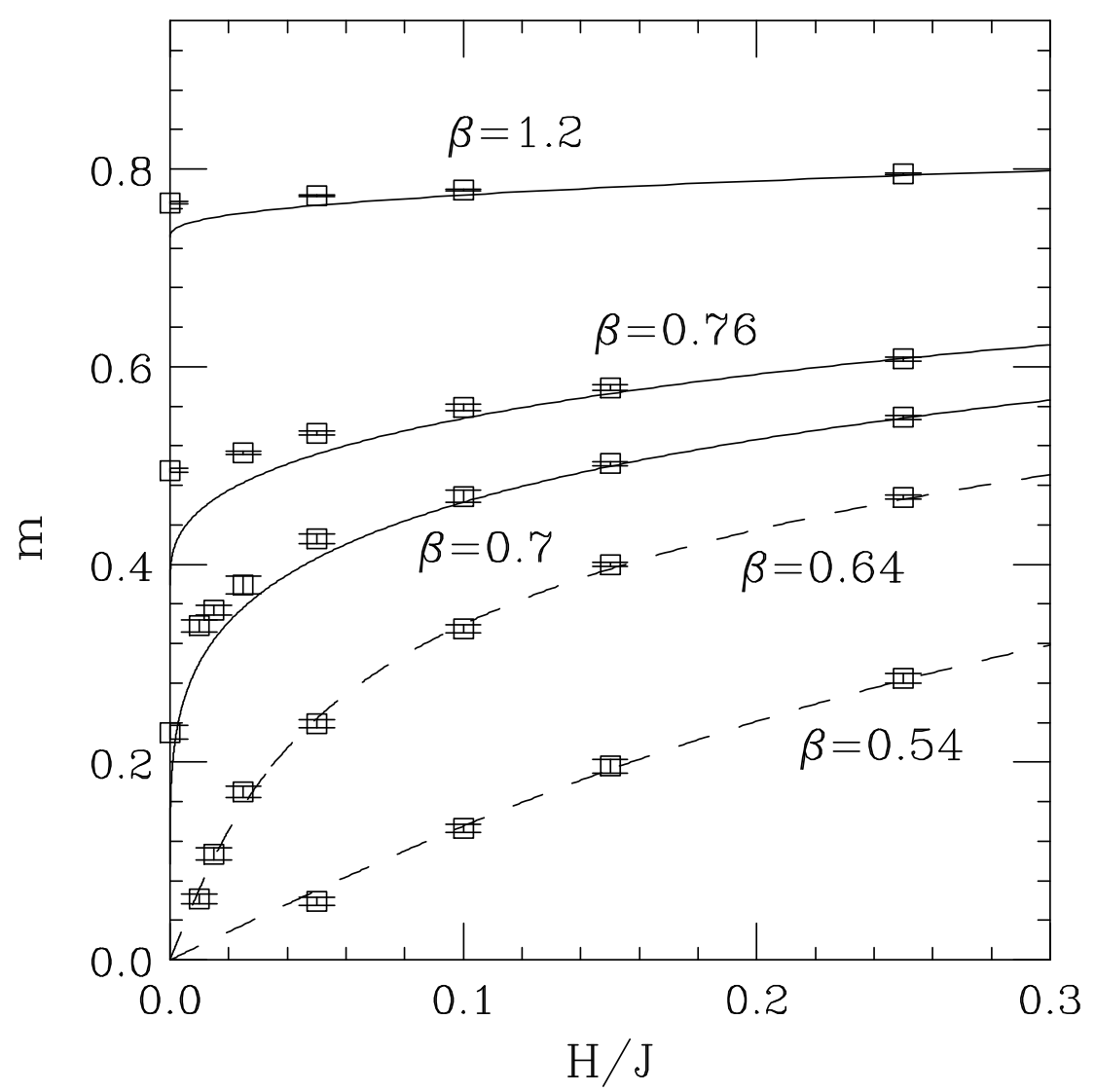

Figure 12: Magnetization isotherms of the classical Heisenberg model (SC lattice). The magnetization $m$ is plotted as a function of the magnetic field $H$ for the values of the inverse temperature $\beta$ shown in the figure. Lines: SCOZA. Squares: MC results [ [4]. Dashed and solid lines refer respectively to super- and sub-critical isotherms. 